\title{
Collection and trade of wild-harvested orchids in Nepal
}

\author{
Abishkar Subedi ${ }^{1,2}$, Bimal Kunwar ${ }^{3}$, Young Choi $^{4}$, Yuntao Dai ${ }^{4}$, Tinde van Andel ${ }^{1}$, Ram P Chaudhary ${ }^{3}$, Hugo J de Boer ${ }^{1,5}$ \\ and Barbara Gravendeel ${ }^{1 *}$
}

\begin{abstract}
Background: Wild orchids are illegally harvested and traded in Nepal for use in local traditional medicine, horticulture, and international trade. This study aims to: 1) identify the diversity of species of wild orchids in trade in Nepal; 2) study the chain of commercialization from collector to client and/or export; 3) map traditional knowledge and medicinal use of orchids; and 4) integrate the collected data to propose a more sustainable approach to orchid conservation in Nepal.
\end{abstract}

Methods: Trade, species diversity, and traditional use of wild-harvested orchids were documented during field surveys of markets and through interviews. Trade volumes and approximate income were estimated based on surveys and current market prices. Orchid material samples were identified to species level using a combination of morphology and DNA barcoding.

Results: Orchid trade is a long tradition, and illegal export to China, India and Hong Kong is rife. Estimates show that 9.4 tons of wild orchids were illegally traded from the study sites during 2008/2009. A total of 60 species of wild orchids were reported to be used in traditional medicinal practices to cure at least 38 different ailments, including energizers, aphrodisiacs and treatments of burnt skin, fractured or dislocated bones, headaches, fever and wounds. DNA barcoding successfully identified orchid material to species level that remained sterile after culturing.

Conclusions: Collection of wild orchids was found to be widespread in Nepal, but illegal trade is threatening many species in the wild. Establishment of small-scale sustainable orchid breeding enterprises could be a valuable alternative for the production of medicinal orchids for local communities. Critically endangered species should be placed on CITES Appendix I to provide extra protection to those species. DNA barcoding is an effective method for species identification and monitoring of illegal cross-border trade.

Keywords: Commercialization, DNA barcoding, Orchids, CITES, Traditional medicine

\section{Introduction}

\section{Medicinal orchids of Nepal}

Orchids are long known for their medicinal value. It is believed that the Chinese were the first to cultivate, describe and use orchids as early as $200 \mathrm{BC}$ [1-3]. In the Indian subcontinent, the Ayurvedic medicinal system uses formulations based on orchid species. Ashtavarga, a group of eight medicinal plants includes four orchid ingredients, Habenaria edgeworthii Hook.f. ex Collet,

\footnotetext{
* Correspondence: barbara.gravendeel@naturalis.nl

${ }^{1}$ Naturalis Biodiversity Center, Sylviusweg 72, P.O. Box 9517, Leiden, The Netherlands

Full list of author information is available at the end of the article
}

H. intermedia, Malaxis acuminata D. Don, and $M$. muscifera $^{\mathrm{a}}$ [3-5].

Wild orchids in Nepal are popularly known by the vernacular name Sungava or Sunakhari, which refers to their shiny yellow pseudobulbs. A total of 377 species belonging to 100 genera have been reported from Nepal, including 12 endemic species [6]. Due to inaccessibility of modern health care facilities, about $80 \%$ of the population of the country still depends on a wide range of locally available medicinal plants for their basic primary healthcare [7]. By 2004, over 590 studies related to ethnobotany in Nepal had been published [8]. Most of these studies lack detailed knowledge on local therapeutic uses of Nepalese orchids or describe very few

\section{Biomed Central}


cases only. In contrast to many other plant families, a comprehensive and detailed study of medicinal orchids in Nepal is still lacking [9].

\section{Trade of wild orchids for medicinal and other commercial} purposes in Nepal

Trade in wild harvested orchids threatens local biodiversity due to overexploitation and habitat destruction [10]. Vaidya et al. [11] reported that around five tons of tubers of Orchis latifolia L. were harvested every year in Nepal to prepare 'Salep' for export at an approximatele value of USD 900 per ton. The export of valuable medicinal species such as Dactylorhiza hatagirea and Gastrodia elata from Nepal to China began in the late 1990s, and continues until today despite attempts to ban trade in these endangered species [12]. Bailes [13] reported in 1985 that in eastern Nepal about 100 trucks with 8 tons capacity each, loaded with wild-collected orchids were shipped to India illegally to prepare various Ayurvedic products. These cases all show that wild orchids from Nepal are popular trade items. The unsustainable use of orchid resources and illegal export of commercially important species causes biodiversity erosion and revenue loss to Nepal [12].

At present, it is difficult to come up with alternative, more sustainable methods to exploit wild Nepalese orchids. Efforts are hampered by three main problems. First of all, many illegally traded orchids cannot be identified to species level as they are often collected and traded sterile. Secondly, expertise in Nepal for artificial propagation of wild orchids is still very limited. Thirdly, surprisingly few studies from Nepal have been published on the trade of wild-collected orchids despite growing concerns on overexploitation and illegal national and cross-border trade $[14,15]$.

This study aims to: i) identify the diversity of species in illegal trade in Nepal; ii) map traditional knowledge and medicinal use of wild orchids; iii) study the chain of commercialization from collector to client and/or export; iv) evaluate the efficacy DNA barcoding for orchid identification; and v) integrate the collected data to propose a more sustainable approach to orchid conservation.

\section{Materials and methods}

\section{Study areas}

Surveys were carried out in three villages in Makwanpur district of central Nepal: Agra, Gogane, and Manahari, and in two markets in the Kathmandu valley: Dakshinkali and Godavari (Figure 1). Surveys were carried out in February-March 2008 and August-November 2009.

\section{Data collection}

Primary data were collected from interviews with local villagers involved in orchid collection, middlemen, vendors, local traders and district forest officials. We used a semistructured questionnaire for the interviews. A detailed inventory of medicinal orchids and their uses in Nepal was prepared by a literature study. Additional information was collected through key informant interviews with local plant healers at the study sites after agreeing to a prior informed consent (PIC) on mutually agreed terms (MAT). A total of 31 people were interviewed.

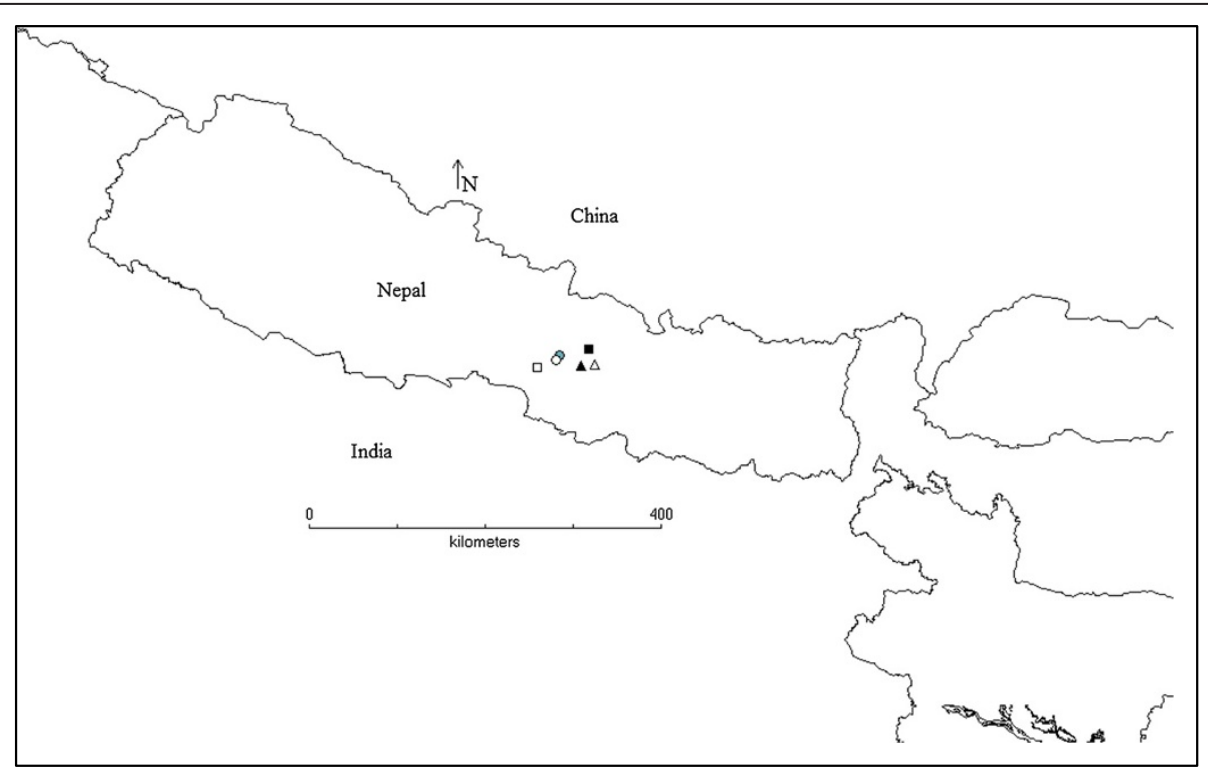

Figure 1 Map showing the study sites in Nepal (• Agra VDC, $\Delta$ Dakshinkali, $\Delta$ Godavari, $\circ$ Gogane VDC, $\square$ Kathmandu, $\square$ Manhari VDC). 


\section{Plant identification}

Wild collected flowering orchids were identified using standard literature [16-18] and cross-referenced with herbarium specimens deposited at Tribhuvan University Central Herbarium (TUCH). Sterile plants were purchased, and small cuttings were cultivated to bloom in an experimental garden in the vicinity of Pokhara for subsequent identification to species level. If no flowering could be initiated, DNA barcoding was applied. Voucher specimens of all orchid species are deposited at TUCH (Table 1).

\section{DNA barcoding of illegally traded orchids}

DNA barcoding is a powerful tool used to control trade in species placed on either CITES Appendix I or II [27-29] and to trace cross-border wildlife crime [30].

Purchased orchid samples that were unidentifiable by morphology, and failed to flower in the experimental garden were selected for DNA barcoding identification at the Laboratory of Plant Systematics, Central Department of Botany, Tribhuvan University, Kathmandu. The following methods and materials were applied: Material was ground to powder in a mortar with liquid nitrogen. Total genomic DNA was extracted from $40-100 \mathrm{mg}$ of powder using the DNeasy Plant mini kit (Qiagen Inc.). Parts of the plastid mat $K$ gene and nuclear nrITS regions were amplified using the primers-19 F; 881R, 731; 2R, and 101 F; 102R, respectively [31]. Polymerase chain reactions were carried out on a PXE 0.2 Thermocycler (Applied Biosystems) in a $25 \mu \mathrm{l}$ volume containing 0.1-50 ng of genomic DNA, 0.1 M of each primer, $10 \mathrm{M}$ of each dNTP, Qiagen PCR buffer (50 mM KCl, $10 \mathrm{mM}$ Tris-HCl pH 8.7, $1.5 \mathrm{mM} \mathrm{MgCl}{ }_{2}$ ) and 1.5 units of Taq DNA polymerase (Qiagen, Inc.). The thermal cycling profile started with a 5 min denaturation step of $94^{\circ} \mathrm{C}$, then comprised 35 cycles each with $20 \mathrm{~s}$ denaturation at $94^{\circ} \mathrm{C}, 20 \mathrm{sec}$ annealing at 48 $51^{\circ} \mathrm{C}$ and $45 \mathrm{~s}$ elongation at $72^{\circ} \mathrm{C}$, and the program ended with $5 \mathrm{~min}$ extension at $72^{\circ} \mathrm{C}$. Amplification products were separated on a $1 \%$ agarose/TAE gel, purified using the QIAquick PCR Purification Kit (Qiagen Inc.) and sequenced on an ABI 3730xl automated sequencer by Macrogen (South Korea) using standard dye-terminator chemistry following the manufacturers protocols (Applied Biosystems). Complementary strands were assembled and edited with Sequencer version 4.01 (Gene Codes Corporation).

NCBI GenBank BLAST searches [32] were used to match DNA sequences generated in this study with those already deposited in the database. Species names were assigned only in cases of a sequence similarity of $100 \%$. DNA sequences generated were submitted to GenBank (accessions JF422074 - JF422082; Table 1).

\section{Results and discussion}

\section{Medicinal orchids of Nepal}

Sixty species were reported to be used for 38 different ailments (Table 1), representing $15 \%$ of the total number of orchids described from Nepal. A recent literature review by Acharya and Rokaya [9] found 82 medicinal orchid species reported from Nepal, 47 of those were also found in this study focusing on a limited area. Hossain [5] in a global literature review on medicinal orchids shows that a total of 129 species are being used for different therapeutic purposes. Eighty-two medicinally used orchids in Nepal imply that the diversity of traditional orchid species in the country is exceptionally high. The high number could be explained by the fact that our study is the first ethnobotanical survey focusing solely on orchids. In addition, the use of DNA barcoding enabled more accurate species identification of sterile material than can be achieved in morphological studies (Table 2).

Acharya and Rokaya [9] recorded 82 medicinal orchid species for Nepal, 34 of which were not recorded in this study, whereas this study recorded 12 additional species. The combined total of both studies comes to 94 medicinally used orchid species. The majority of these are epiphytes, a fourth is terrestrial, and just a few are lithophytes. Coelogyne, Dendrobium, Cymbidium, Bulbophyl lum, Habenaria, Malaxis and Pholidota are the genera of which most species are being used as traditional medicines. Other reported uses of these medicinal orchids are fodder (25), vegetables (6) and ritual and ceremonial uses (6) [10].

The most common vernacular names for orchids are Sungava and Sunakhari. In addition 23 vernacular names for orchids were recorded to be used by local communities in different parts of Nepal (Table 1). Among these most common are: Thuur or Thurjo (moss-like plants growing on tree trunks), Parajivi (parasitic plant), Bankera (pseudobulbs shaped like a wild banana), Banaduwa (gingerlike), Chandigava (silver-coloured flowers), Shaktigumba (pseudobulbs providing energy) and Chadephul (flowers inducing vomiting). The vernacular names reflect the vast knowledge of local communities with regard to orchid growing habits, habitats and their potential uses.

Major reported local uses include aphrodisiacs, energizers, and treatments of skin burns, fractured or dislocated bones, headaches, fever, and wounds. Other uses include insect repellent, blood purifier, skin fungi, snake and scorpion bite antidote, inducement of abortions, and recovery from childbirth. Orchids are mainly used as paste, powder or juice, solely or mixed with milk, honey or wheat flour. Orchid extracts are either consumed orally or applied externally. A widespread local use of Coelogyne is to eat freshly cut slices of the pseudobulb as a thirst quencher. 
Table 1 Orchids reported in traditional medicine and commercial trade in Nepal

\begin{tabular}{|c|c|c|c|c|c|}
\hline Scientific name voucher number ${ }^{a}$ & Local name & $\begin{array}{l}\text { Parts } \\
\text { used }^{b}\end{array}$ & Traditional use & Reference & $\begin{array}{l}\text { Commercial } \\
\text { trade }\end{array}$ \\
\hline $\begin{array}{l}\text { Acampe praemorsa (Roxb.) Blatt. \& } \\
\text { McCann (syn. Acampe papillosa } \\
\text { (Lindl.) Lindl.) Subedi } 170\end{array}$ & $\begin{array}{l}\text { Parajivi, Rasna } \\
\text { (Sanskrit) }\end{array}$ & Ra & $\begin{array}{l}\text { Powder used in treating rheumatism and } \\
\text { for cooling effect. }\end{array}$ & This study & Medicinal \\
\hline Aerides multiflora Roxb. Kunwar 101 & Parajivi, Thuur & Lf & Powder used in tonic preparation. & This study & $\begin{array}{l}\text { Floricultural, } \\
\text { medicinal }\end{array}$ \\
\hline Aerides odorata Lour. Subedi 172 & Parajivi & Lf & Paste of leaves used externally to treat wounds. & This study & $\begin{array}{l}\text { Floricultural, } \\
\text { medicinal }\end{array}$ \\
\hline $\begin{array}{l}\text { Brachycorythis obcordata (Lindl.) } \\
\text { Summerh. Subedi } 150\end{array}$ & Gamdol & $\mathrm{Tb}$ & Powder mixed with milk and consumed as tonic. & [19] & Medicinal \\
\hline $\begin{array}{l}\text { Bulbophyllum careyanum (Hook.) } \\
\text { Spreng. Subedi } 220\end{array}$ & $\begin{array}{l}\text { Banharchul, } \\
\text { Thuur, Parajivi }\end{array}$ & $\mathrm{Lf}, \mathrm{Pb}$ & $\begin{array}{l}\text { Fresh pulp of pseudobulbs externally applied } \\
\text { to burns. Powder of leaves used with honey to } \\
\text { induce abortions within } 3 \text { months of pregnancy } \\
\text { and stimulate recovery from childbirth. }\end{array}$ & $\begin{array}{l}{[10], \text { This }} \\
\text { study }\end{array}$ & Medicinal \\
\hline $\begin{array}{l}\text { Bulbophyllum leopardinum (Wall.) } \\
\text { Lindl. ex Wall. Subedi } 221\end{array}$ & Thuur, Parajivi & $\mathrm{Lf}, \mathrm{Pb}$ & Fresh pulp or juice externally applied to burns. & This study & Medicinal \\
\hline $\begin{array}{l}\text { Bulbophyllum odoratissimum (Sm.) } \\
\text { Lindl. ex Hook. f. Subedi } 370\end{array}$ & Thurjo & Ep & $\begin{array}{l}\text { Powder used in treating tuberculosis, } \\
\text { chronic inflammation and fractures. }\end{array}$ & [20] & Medicinal \\
\hline $\begin{array}{l}\text { Calanthe sylvatica (Thouars) Lindl. } \\
\text { Subedi } 153\end{array}$ & Pakha phul & $\mathrm{Fl}$ & Juice applied to stop nosebleeds. & [11] & $\begin{array}{l}\text { Floricultural, } \\
\text { medicinal }\end{array}$ \\
\hline Calanthe plantaginea Lindl. Kunwar 120 & Ban aduwa & Rz & $\begin{array}{l}\text { Dry powder consumed with milk as tonic } \\
\text { and as aphrodisiac. }\end{array}$ & This study & $\begin{array}{l}\text { Floricultural, } \\
\text { medicinal }\end{array}$ \\
\hline Calanthe puberula Lindl. Subedi 223 & Ban aduwa & $\mathrm{Rz}$ & $\begin{array}{l}\text { Dry powder consumed with milk as tonic } \\
\text { and as aphrodisiac. }\end{array}$ & This study & $\begin{array}{l}\text { Floricultural, } \\
\text { medicinal }\end{array}$ \\
\hline Coelogyne corymbosa Lindl. Subedi 375 & Chadigava & $\mathrm{Pb}$ & $\begin{array}{l}\text { Paste applied to the forehead to relieve headaches, } \\
\text { fresh juice applied to burns as analgesic. }\end{array}$ & {$[7,11,21]$} & $\begin{array}{l}\text { Floricultural, } \\
\text { medicinal }\end{array}$ \\
\hline Coelogyne cristata Lindl. Subedi 224 & $\begin{array}{l}\text { Chandigava, } \\
\text { Bankera }\end{array}$ & $\mathrm{Pb}$ & $\begin{array}{l}\text { Freshly collected paste or juice consumed to relieve } \\
\text { headaches, fever and for indigestion. Pulp applied to } \\
\text { burnt skin. Juice also applied to skin boils and } \\
\text { wounded hooves of cattle. }\end{array}$ & $\begin{array}{l}{[1,10,21,22]} \\
\text { This study }\end{array}$ & $\begin{array}{l}\text { Floricultural, } \\
\text { medicinal }\end{array}$ \\
\hline Coelogyne fimbriata Lindl. Subedi 225 & $\begin{array}{l}\text { Jiwanti } \\
\text { (Sanskrit) }\end{array}$ & $\mathrm{Pb}$ & Powder used in tonic preparation. & This study & $\begin{array}{l}\text { Floricultural, } \\
\text { medicinal }\end{array}$ \\
\hline Coelogyne flaccida Lindl. Subedi 301 & Chadigava & $\mathrm{Pb}$ & $\begin{array}{l}\text { Paste applied externally or consumed to relieve } \\
\text { frontal headaches. Juice taken for indigestion. }\end{array}$ & {$[7,21]$} & $\begin{array}{l}\text { Floricultural, } \\
\text { medicinal }\end{array}$ \\
\hline Coelogyne fuscescens Lindl. Subedi 312 & Bankera & $\mathrm{Pb}$ & $\begin{array}{l}\text { Paste applied externally or consumed to relieve } \\
\text { headaches, fever and stomach ache. Paste } \\
\text { applied externally on burns. }\end{array}$ & This study & $\begin{array}{l}\text { Floricultural, } \\
\text { medicinal }\end{array}$ \\
\hline $\begin{array}{l}\text { Coelogyne nitida (Wall. ex D.Don) } \\
\text { Lindl. Subedi } 226\end{array}$ & $\begin{array}{l}\text { Banlasun, } \\
\text { Thuur }\end{array}$ & $\mathrm{Pb}$ & $\begin{array}{l}\text { Paste consumed against headaches and fever. } \\
\text { Paste applied externally on burns. }\end{array}$ & This study & $\begin{array}{l}\text { Floricultural, } \\
\text { medicinal }\end{array}$ \\
\hline Coelogyne prolifera Lindl. Subedi 227 & Thuur & $\mathrm{Pb}$ & $\begin{array}{l}\text { Paste consumed against headaches and fever. } \\
\text { Paste applied externally on burns. }\end{array}$ & {$[10]$} & $\begin{array}{l}\text { Floricultural, } \\
\text { medicinal }\end{array}$ \\
\hline $\begin{array}{l}\text { Coelogyne stricta (D.Don) Schltr. } \\
\text { Subedi } 314\end{array}$ & Banpyaj & $\mathrm{Pb}$ & Paste applied externally against headaches and fever. & This study & $\begin{array}{l}\text { Floricultural, } \\
\text { medicinal }\end{array}$ \\
\hline $\begin{array}{l}\text { Crepidium acuminatum (D.Don) } \\
\text { Szlach. (syn. Malaxis acuminata D. Don) } \\
\text { Subedi } 321\end{array}$ & $\begin{array}{l}\text { Gachno, } \\
\text { Gavndamala }\end{array}$ & $\mathrm{Ra}, \mathrm{Pb}$ & $\begin{array}{l}\text { Powder of roots used against burning sensations, } \\
\text { to treat fever and to stop bleeding. }\end{array}$ & This study & Medicinal \\
\hline $\begin{array}{l}\text { Cymbidium aloiflolium (L.) } \\
\text { Sw. Subedi } 228\end{array}$ & $\begin{array}{l}\text { Banharchul, } \\
\text { Kamaru, Harjor }\end{array}$ & Ep & $\begin{array}{l}\text { Dried powder used as tonic against diarrhea. } \\
\text { Fresh paste applied externally over fractured } \\
\text { or dislocated bones. }\end{array}$ & {$[10,23]$} & $\begin{array}{l}\text { Floricultural, } \\
\text { medicinal }\end{array}$ \\
\hline Cymbidium elegans Lindl. Kunwar 123 & Thuur & $\mathrm{Ra}, \mathrm{Pb}$ & $\begin{array}{l}\text { Fresh juice of pseudobulb consumed to relieve fever. } \\
\text { Boiled root juice fed to livestock suffering from cold. }\end{array}$ & {$[21,22]$} & $\begin{array}{l}\text { Floricultural, } \\
\text { medicinal }\end{array}$ \\
\hline Cymbidium iridioides D. Don Subedi 315 & Thuur & $\mathrm{Pb}, \mathrm{Lf}$ & $\begin{array}{l}\text { Powder of pseudobulb consumed as tonic. Leaf juice } \\
\text { applied externally to stimulate blood clotting in } \\
\text { deep wounds. }\end{array}$ & $\begin{array}{l}\text { [11], This } \\
\text { study }\end{array}$ & $\begin{array}{l}\text { Floricultural, } \\
\text { medicinal }\end{array}$ \\
\hline
\end{tabular}


Table 1 Orchids reported in traditional medicine and commercial trade in Nepal (Continued)

\begin{tabular}{|c|c|c|c|c|c|}
\hline Cypripedium himalaicum Rolfe Kunwar 124 & Khujukpa & Ep & $\begin{array}{l}\text { Powder and juice consumed for urine retention, } \\
\text { against kidney stones, heart disease, chest } \\
\text { disorders and coughs. }\end{array}$ & [24] & Medicinal \\
\hline $\begin{array}{l}\text { Dactylorhiza hatagirea (D.Don) } \\
\text { Soo Kunwar } 103\end{array}$ & $\begin{array}{l}\text { Paanchaunle, } \\
\text { Hatajadi }\end{array}$ & $\mathrm{Tb}$ & $\begin{array}{l}\text { Paste consumed against fever. Powder used topically } \\
\text { as hemostatic, or to heal fractures. Decoction } \\
\text { consumed against intestinal pain. Tuber, eaten raw } \\
\text { or as tonic, or mixed with honey or milk used } \\
\text { as stimulant. }\end{array}$ & {$[7,23]$} & Medicinal \\
\hline $\begin{array}{l}\text { Dendrobium amoenum Wall. } \\
\text { ex Lindl. Subedi } 400\end{array}$ & Thuur & $\mathrm{Pb}$ & $\begin{array}{l}\text { Fresh paste applied topically on burnt skin } \\
\text { and dislocated bones. }\end{array}$ & This study & Medicinal \\
\hline $\begin{array}{l}\text { Dendrobium densiflorum Lindl. } \\
\text { Subedi } 316\end{array}$ & Sungava & $\mathrm{Pb}$ & Fresh pulp applied to boils and pimples. & {$[21,22]$} & $\begin{array}{l}\text { Floricultural, } \\
\text { medicinal }\end{array}$ \\
\hline Dendrobium eriiflorum Griff. Kunwar 104 & Thurjo & $\mathrm{Pb}$ & $\begin{array}{l}\text { Paste mixed with wheat flour and applied on } \\
\text { dislocated or fractured bones. Dried powder used } \\
\text { as tonic. }\end{array}$ & This study & $\begin{array}{l}\text { Floricultural, } \\
\text { medicinal }\end{array}$ \\
\hline $\begin{array}{l}\text { Dendrobium heterocarpum Wall. } \\
\text { ex Lindl. Subedi } 317\end{array}$ & Thuur & $\mathrm{Pb}$ & $\begin{array}{l}\text { Paste mixed with wheat flour and applied } \\
\text { on fractured or dislocated bones. }\end{array}$ & This study & $\begin{array}{l}\text { Floricultural, } \\
\text { medicinal }\end{array}$ \\
\hline $\begin{array}{l}\text { Dendrobium longicornu Lindl. Subedi } \\
401\end{array}$ & Kause & $\mathrm{Ra}, \mathrm{Pb}$ & $\begin{array}{l}\text { Juice of stems is consumed against fever. Boiled } \\
\text { root fed to livestock suffering from coughs. }\end{array}$ & [7] & $\begin{array}{l}\text { Floricultural, } \\
\text { medicinal }\end{array}$ \\
\hline $\begin{array}{l}\text { Dendrobium transparens Wall. } \\
\text { ex Lindl. Subedi } 402\end{array}$ & Parajivi, Thuur & $\mathrm{Pb}$ & Paste used on fractured or dislocated bones. & [10] & $\begin{array}{l}\text { Floricultural, } \\
\text { medicinal }\end{array}$ \\
\hline $\begin{array}{l}\text { Dienia cylindrostachya Lindl. (syn. Malaxis } \\
\text { cylindrostachya (Lindl.) Kuntze) Kunwar } 132\end{array}$ & & $\mathrm{~Pb}$ & Powder used as tonic. & This study & Medicinal \\
\hline $\begin{array}{l}\text { Epipactis helleborine (L.) Crantz Kunwar } \\
133\end{array}$ & & $\mathrm{Ra}$ & Juice consumed to cure insanity and gout. & [11] & Medicinal \\
\hline $\begin{array}{l}\text { Eria spicata (D.Don) Hand.-Mazz. Subedi } \\
403\end{array}$ & Parajivi & $\mathrm{Pb}$ & $\begin{array}{l}\text { Powder consumed during stomach ache, } \\
\text { paste applied externally against headaches. }\end{array}$ & [11] & Medicinal \\
\hline $\begin{array}{l}\text { Eulophia dabia (D.Don) Hochr. Kunwar } \\
134\end{array}$ & Hatti paila & $\mathrm{Rz}$ & $\begin{array}{l}\text { Powder consumed against coughs and heart } \\
\text { trouble, also used as tonic and appetizer. }\end{array}$ & {$[1,11]$} & Medicinal \\
\hline $\begin{array}{l}\text { Eulophia spectabilis (Dennst.) Suresh } \\
\text { (syn. Eulophia nuda Lindl.) Kunwar } 135\end{array}$ & Amarkand & $\mathrm{Tb}$ & $\begin{array}{l}\text { Powder used against worm infestation, scrofula, } \\
\text { blood disorders, bronchitis and as appetizer. }\end{array}$ & [11] & Medicinal \\
\hline $\begin{array}{l}\text { Flickingeria fugax (Rchb.f.) } \\
\text { Seidenf. Kunwar } 140\end{array}$ & Jiwanti & Ep & $\begin{array}{l}\text { Powder used as tonic against general debility } \\
\text { and as stimulant. }\end{array}$ & This study & Medicinal \\
\hline $\begin{array}{l}\text { Flickingeria macraei (Lindl.) } \\
\text { Seidenf. Subedi } 319\end{array}$ & Jiwanti & Ep & $\begin{array}{l}\text { Paste used against snake bites, general debility, } \\
\text { as stimulant and demulcent. }\end{array}$ & {$[1,25]$} & Medicinal \\
\hline Gastrodia elata Blume Subedi 421 & & $\mathrm{~Tb}$ & $\begin{array}{l}\text { Dried powder used as tonic and for } \\
\text { treating headaches. }\end{array}$ & [11] & Medicinal \\
\hline Gymnadenia orchidis Lindl. Kunwar 141 & & $\mathrm{~Tb}$ & $\begin{array}{l}\text { Powder used to treat gastric, urine and liver } \\
\text { disorders. }\end{array}$ & [11] & Medicinal \\
\hline Habenaria intermedia D.Don Subedi 422 & Riddhi & $R a, L f$ & Powder used for blood diseases. & [3] & Medicinal \\
\hline Habenaria pectinata D.Don Kunwar 141 & Seto musli & Tb, Lf & $\begin{array}{l}\text { Leaf juice applied on snake bites. Tuber used } \\
\text { against arthritis. }\end{array}$ & [3] & Medicinal \\
\hline $\begin{array}{l}\text { Luisia trichorrhiza (Hook.) } \\
\text { Blume Subedi } 320\end{array}$ & Arjona & Lf & Paste applied externally to treat muscular pain. & [11] & Medicinal \\
\hline $\begin{array}{l}\text { Luisia tristis (G. Forst.) Hook.f. } \\
\text { (syn Luisia zeylanica Lindl.) Subedi } 423\end{array}$ & Bori jhaar & Ep & Juice used for treating chronic wounds. & [11] & Medicinal \\
\hline $\begin{array}{l}\text { Malaxis muscifera (Lindl.) } \\
\text { Kuntze Kunwar } 142\end{array}$ & Jivaka & $\mathrm{Pb}$ & $\begin{array}{l}\text { Paste applied during diathesis, burning sensation, } \\
\text { fever, on sores and as tonic. }\end{array}$ & This study & Medicinal \\
\hline Otochilus albus Lindl. Subedi 370 & Aankhle laharo & Ep & Powder used as tonic. & This study & Medicinal \\
\hline $\begin{array}{l}\text { Otochilus lancilabius Seidenf. Kunwar } \\
107\end{array}$ & Aankhle laharo & Ep & Paste applied to fractured and dislocated bones. & [6] & Medicinal \\
\hline $\begin{array}{l}\text { Papilionanthe teres (Roxb.) } \\
\text { Schltr. Subedi } 424\end{array}$ & Harjor, Thurjo & $\mathrm{Pb}, \mathrm{Lf}$ & Paste externally applied to treat dislocated bones. & {$[7]$} & Medicinal \\
\hline Pholidota articulata Lindl. Subedi 368 & Hadjor & Ep & $\begin{array}{l}\text { Paste applied on fractured bones and consumed } \\
\text { as tonic. }\end{array}$ & This study & $\begin{array}{l}\text { Floricultural, } \\
\text { medicinal }\end{array}$ \\
\hline
\end{tabular}


Table 1 Orchids reported in traditional medicine and commercial trade in Nepal (Continued)

\begin{tabular}{|c|c|c|c|c|c|}
\hline Pholidota imbricata Lindl. Subedi 367 & $\begin{array}{l}\text { Thurjo, } \\
\text { Patharkera }\end{array}$ & $\mathrm{Pb}$ & $\begin{array}{l}\text { Paste consumed to relieve fever and } \\
\text { powder as tonic. }\end{array}$ & This study & Medicinal \\
\hline Pholidota pallida Lindl. Subedi 369 & $\begin{array}{l}\text { Thurjo, } \\
\text { Patharkera }\end{array}$ & $\mathrm{Rh}, \mathrm{Pb}$ & $\begin{array}{l}\text { Paste used to relieve fever, powder to induce } \\
\text { sleep and to cure abdominal pain, juice used for } \\
\text { navel pain. }\end{array}$ & This study & $\begin{array}{l}\text { Floricultural, } \\
\text { medicinal }\end{array}$ \\
\hline $\begin{array}{l}\text { Platanthera edgeworthii } \\
\text { (Hook.f. ex Collett) R.K.Gupta } \\
\text { (syn. Habenaria edgeworthii Hook.f. } \\
\text { ex Collett) Kunwar } 145\end{array}$ & Riddhi & Rh, Lf & $\begin{array}{l}\text { Powder or paste consumed to cure blood diseases } \\
\text { and for cooling. }\end{array}$ & {$[3]$} & Medicinal \\
\hline Pleione humilis (Sm.) D.Don Kunwar 108 & Shaktigumba & $\mathrm{Pb}$ & $\begin{array}{l}\text { Paste applied on cuts and wounds. Powder used } \\
\text { as tonic. }\end{array}$ & {$[7,21]$} & $\begin{array}{l}\text { Floricultural, } \\
\text { medicinal }\end{array}$ \\
\hline Pleione praecox (Sm.) D.Don Kunwar 109 & Shaktigumba & $\mathrm{Pb}$ & $\begin{array}{l}\text { Dried powder consumed with milk as tonic and } \\
\text { energizer. Paste externally applied on cuts } \\
\text { and wounds. }\end{array}$ & $\begin{array}{l}{[22], \text { This }} \\
\text { study }\end{array}$ & $\begin{array}{l}\text { Floricultural, } \\
\text { medicinal }\end{array}$ \\
\hline Rhynchostylis retusa (L.) Blume & $\begin{array}{l}\text { Chadephuul, } \\
\text { Dhogegava }\end{array}$ & Ep & $\begin{array}{l}\text { Juice of roots applied to cuts and wounds. Leaf } \\
\text { powder used to cure rheumatic diseases. Dried } \\
\text { flowers as insect repellent and to induce vomiting. }\end{array}$ & {$[1,7,10,22]$} & $\begin{array}{l}\text { Floricultural, } \\
\text { medicinal }\end{array}$ \\
\hline Satyrium nepalense D.Don & Mishri, Thamni & $\mathrm{Tb}$ & $\begin{array}{l}\text { Dried tubers consumed as tonic against dysentery. } \\
\text { Juice consumed against fever and applied on cuts } \\
\text { and wounds. }\end{array}$ & {$[7,21]$} & Medicinal \\
\hline $\begin{array}{l}\text { Spiranthes sinensis (Pers.) } \\
\text { Ames Subedi } 451\end{array}$ & & $\mathrm{~Tb}$ & $\begin{array}{l}\text { Powder consumed against headaches as tonic } \\
\text { and energizer. }\end{array}$ & {$[19,26]$} & Medicinal \\
\hline Thunia alba (Lindl.) Rchb.f. & Golaino & Ep & Paste used on fractured bones. & {$[25]$} & Medicinal \\
\hline Vanda cristata Wall. ex Lindl. Subedi 201 & $\begin{array}{l}\text { Vhagute Phul, } \\
\text { Thuur }\end{array}$ & $R a, L f$ & $\begin{array}{l}\text { Root paste applied to boils and to treat dislocated } \\
\text { bones. Leaf powder used as expectorant, paste } \\
\text { applied to cuts and wounds. }\end{array}$ & {$[7,21]$} & $\begin{array}{l}\text { Floricultural, } \\
\text { medicinal }\end{array}$ \\
\hline $\begin{array}{l}\text { Vanda tessellata (Roxb.f.) Hook.ex G.Don } \\
\text { (syn. Vanda roxburghii R.Br.) Subedi } 467\end{array}$ & Parajivi, Rasna & $\mathrm{Ra}, \mathrm{Lf}$ & $\begin{array}{l}\text { Root used as antidote for scorpion stings, and } \\
\text { remedy of bronchitis and rheumatism. } \\
\text { Paste of leavesused to treat fevers. }\end{array}$ & {$[3]$} & $\begin{array}{l}\text { Floricultural, } \\
\text { medicinal }\end{array}$ \\
\hline $\begin{array}{l}\text { Zeuxine strateumatica (L.) } \\
\text { Schltr. Subedi } 200\end{array}$ & Kansjhar & Rt & Dry powder used as tonic. & This study & Medicinal \\
\hline
\end{tabular}

${ }^{\mathrm{a}}$ Vouchers are deposited at $\mathrm{TUCH} ;{ }^{\mathrm{b}} \mathrm{Fl}$, flowers; Lf, leaves; Pb, pseudobulb; Ra, roots; Rz, rhizome; Tb, tubers; Ep, entire plant.

\section{Wild orchid species in trade}

From the total of 60 species of wild orchids recorded as traded from the study sites, 28 species were exported both for medicinal and floricultural purposes, and 32 species for medicinal purposes only. Multiple use-values exacerbate the threat of overexploitation for these species. For medicinal purposes, species belonging to the genera Acampe, Aerides, Coelogyne, Crepidium, Dactylorhiza, Dendrobium, Gastrodia, Eulophia, Flickingeria, Otochilus,
Pholidota, Satyrium and Vanda are most heavily exploited based on the number of times these were cited by the respondents. Acampe praemorsa, Aerides multiflora, Bulbophyllum careyanum, Coelogyne cristata, Co. nitida, Crepidium acuminatum, Dactylorhiza hatagirea, Den drobium aphyllum, De. crepidatum, De. eriiflorum, De. moschatum, Eulophia spectabilis, Flickingeria fugax, Gas trodia elata, Otochilus albus, Pholidota pallida, Ph. imbricata and Vanda cristata are the most wanted species

Table 2 DNA barcoding of sterile medicinal orchids

\begin{tabular}{|c|c|c|c|c|}
\hline \multirow{2}{*}{$\begin{array}{l}\text { Medicinal orchid } \\
\text { Scientific name Voucher number }\end{array}$} & \multicolumn{4}{|c|}{ DNA barcoding } \\
\hline & Marker & Voucher sequence & Reference sequence & Citation for reference sequence \\
\hline \multirow[t]{2}{*}{ Coelogyne cristata Lindl. Subedi 224} & nrlTs & & & [31] \\
\hline & matK & JF422077 & AF302707 & \\
\hline \multirow[t]{2}{*}{ Coelogyne fimbriata Lindl. Subedi 225} & nrlTS & JF422074 & AF302745 & {$[31]$} \\
\hline & matK & JF422078 & AF302710 & \\
\hline \multirow[t]{2}{*}{ Coelogyne stricta (D. Don) Schltr.; Subedi 314} & nrlTS & JF422075 & AF302757 & {$[31]$} \\
\hline & matk & $J F 422079$ & AF302722 & \\
\hline \multirow[t]{2}{*}{ Pleione praecox D. Don Kunwar 109} & nrlTs & JF422076 & AF461491 & {$[33]$} \\
\hline & matK & JF422082 & AF503742 & \\
\hline
\end{tabular}


for rituals. Coelogyne cristata, Co. flaccida, Co. nitida, Cymbidium iridioides, Dendrobium densiflorum and Vanda cristata are most widely exploited as cut flowers.

\section{Orchid collectors and collecting practices}

Collecting wild orchids was predominantly done by local youths, women and children, and a total of 42 collectors were recorded across the study sites. At Dakshinkali, at least 18 local collectors were involved in orchid collection, supplying the 10 local vendors with orchids. The vendors themselves were sometimes also involved in collecting wild orchids. Some local collectors reported to have been involved in orchid collection and selling for more than 25 years.

Medicinal orchids were usually harvested from December up to April with a peak period from January to March. For floriculture, the collection period was found to be throughout the year depending on the availability of flowering individuals. Collectors reported to search far and wide for orchids, frequently traveling more than $10 \mathrm{~km}$ on foot through the forest. Epiphytic orchids growing high up in tree canopies were collected by felling the trees if feasible, and preferentially collected in clumps. Terrestrial orchids were collected by unearthing the tubers to take the entire plant.
Collection of wild orchids usually started once a purchase order was received from middlemen. These persons usually stayed nearby orchid collection sites throughout the collection period. Sometimes, the collectors received advance payments. The middlemen usually came from distant districts or even abroad, and provided printed photographs of desired species or small samples of life orchids, and asked collectors to collect similar-looking plants. An example of such a photograph was retrieved from a middleman who received it from international traders based in Hong Kong (Figure 2E). Local people collected all orchids found, also when these did not resemble the species on the photographs provided by the middlemen. None of the orchids collected were discarded at the selling points. Most collectors spent an average of 5-6 $\mathrm{h}$ per day in the forest. They carried the orchids in bamboo baskets (Figrue 2A-D) or in jute sacks to the nearest selling points. Over the past 15 years, large-scale orchid collection in Nepal has increased year-on-year based on the traded volumes cited by the respondents.

\section{Wild orchids market outlets}

Dakshinkali, $22 \mathrm{~km}$ from Kathmandu, is the center of wild orchid trade in Nepal, and orchids have been sold for over 25 years. Dakshinkali has at least 10 vendors that are
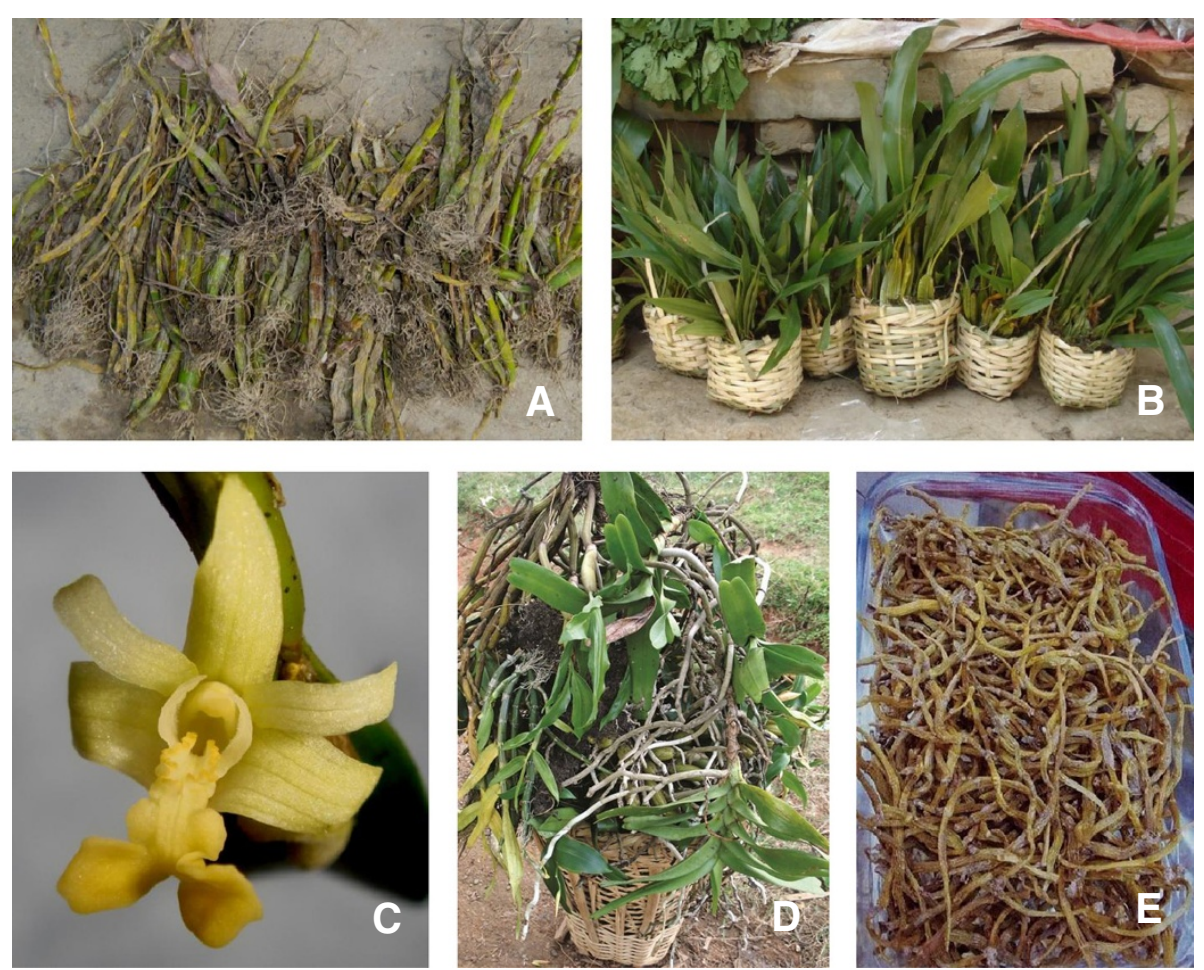

Figure 2 Trade in illegally collected medicinal orchids in Nepal. 2A. Dendrobium erifflorum (photograph: B. Kunwar). 2B. Coelogyne species in market outlets (photograph: A. Subedi). 2C. Flickingeria fugax (photograph: A. Subedi). 2D. Aerides and Dendrobium species in traditional bamboo baskets (photograph: B.B. Raskoti). 2E. Dendrobium eriiflorum originating from Nepal in Hong Kong supermarkets (photograph: Anonymous). 
specialized in wild orchid trade. Another significant trade hub is Godavari, near Kathmandu, but orchid trade here has gradually declined over the last five years. Dakshinkali is famous for its historic Hindu Kali temple, and every year up to 400.000 pilgrims visit this temple, and purchase wild orchids, which play an important role in ceremonial rituals. Many hotel owners in Kathmandu buy wild orchids at Dakshinkali. These orchids can easily be recognized by their traditional woven bamboo baskets that are specially made for the purpose of selling wild orchids, and not found elsewhere in Nepal.

The east-west highway of the tropical part of central Nepal is another very active site for orchid trade. No fixed orchid selling locations are present here, but every year, the middlemen and/or local traders inform the collectors where the orchids should be brought. At these transitory trade points, the orchids are weighed and traded, with large volumes loaded onto trucks or tractors and transported illegally to India or China.

\section{Wild orchids trade volume and local income}

The peak season for orchid trade at Dakshinkali is from July to October. In this period in the year 2008-2009, each live orchid vendor sold an average of 15-20 pots per day, which averages to $2-2.5 \mathrm{~kg}$ of orchids. Extrapolated to yearly trade per vendor this averages to 4.4 tons of orchids per year $(2.25 \mathrm{~kg} \times 17.5$ pots $\times 7$ days $\times 16$ weeks $)$. The vendors sold both vegetative and flowering orchids, but the latter fetched the highest prices. Popular species such as Dendrobium densiflorum, Coelogyne cristata, Cymbidium iridoides and Cymbidium erythraeum traded at the highest values. The price of orchids for floricultural purposes was highly variable and fluctuating, but averaged USD 1.0-1.5 per pot. This average price allows us to make a rough estimate of the annual income per vendor from orchid trade: 17.5 pots $\times 1.25$ USD $\times 7$ days $\times$ 16 weeks $=2450$ USD.

Local medicinal orchid traders and middlemen reported that orchid trade had declined recently due to the arrest of a number of illegal traders. Collectors were reported to earn an average of USD 2 per $\mathrm{kg}$ for medicinal orchids with prices varying between USD 1.5-2.5 depending on the species and quality of the orchids. Based on the interviews we estimate an annual trade of 5 tons for 2008-2009, yielding a combined annual total for Dakshinkali of 9.4 tons of wild orchids for that year.

Detailed export prices of wild orchids collected at the study sites could not be assessed since the traders refused to provide these data. One trader informed us that processed Dendrobium eriiflorum sold for 10,000 Hong Kong dollars ( 1300 USD) per kg. This is in line with the general conception that wild orchids from
Nepal fetch higher prices internationally than on the domestic market.

\section{Legal and illegal trade destinations of Nepalese orchids}

Interviews with collectors, middlemen and local traders revealed that most of the wild orchids collected in Nepal are exported to India and China, and occasionally to Hong Kong. None of the actors involved had received permission from local authorities. The local traders mostly exported raw or occasionally semi-processed, dried and cleaned, products. Our findings support previous reports about illegal trade in Nepalese orchids [13,15]. Shakya et al. [14] reported that wild orchids from Nepal were exported to European countries for floricultural purposes, with none of the exported species grown at nurseries. Nepalese newspapers frequently report cases in which orchid smugglers are arrested with huge quantities of wild orchids for export to China.

\section{Discussion and conclusions}

\section{Sustainable use of medicinal orchids}

Collection and use of wild orchids of Nepal is deeply engrained in the traditional livelihoods of local communities. They form an important part of the traditional health care system and provide a substantial income to subsistence farmers. An increasing number of species are now illegally traded in bulk volumes to some of the most rapidly growing economies in the region, China, India and Hong Kong. This illegal trade creates a severe threat to wild orchid populations in Nepal [34], urging development of alternative strategies for sustainable exploitation. We advocate development of sustainable orchid enterprises focusing on medicinal orchid species grown from cuttings and seed. Cultivated of orchids for raw ingredients of herbal medicine is a niche in the international orchid market that is still relatively undeveloped, and deserves further exploration $[2,35,36]$.

Artificial propagation of orchids has the potential to reduce illegal collecting in the wild through wider availability of stock material, and can also provide large numbers of plants within a short period of time. Artificially propagated plants often have the advantage of being more vigorous than wild collected stock, have a higher survival rate and contain higher contents of compounds with pharmacological effects $[37,38]$. The establishment of a sustainable national orchid industry based on low cost in vitro propagation could be beneficial to the conservation of endangered orchids, and for several species of wild-collected Nepali orchids these techniques have already been developed [39-41]. The potential disadvantage of undercutting local collectors and traders is that as their livelihoods are jeopardized, they are forced to diverge into other sources of supplementary income, such as the collection of other medicinal plant species. 


\section{Policies for protection of wild orchids in Nepal}

All wild orchids of Nepal are protected under Appendix II of the Convention on International Trade in Endangered species of Wild Fauna and Flora (CITES). The Forest Act 1993, and Forest Regulations 1995, and amendment in 2001 specified all orchids in Nepal as protected. However, contradicting its own policies, the Government of Nepal published a notification on April 14th, 2008 permitting collection of wild orchids for trade. The absence of clear guidelines on sustainable harvesting and weak enforcement of policies could explain the recent increase in illegal trade in orchids [22].

\section{DNA barcoding for identification of sterile orchids}

Sterile plant parts sold at local markets can be identified to species level using DNA barcoding. DNA barcoding is increasingly applied for plant species identification $[42,43]$. This method can both provide the taxonomic identity of samples analyzed, and - if the markers employed are sensitive enough - elucidate the geographical origin of the collected species [28]. For the former scenario, DNA barcoding is ever-increasingly facilitating monitoring trade of CITES-listed species [27]. For the latter, the method is more and more used to trace and substantiate crossborder wildlife crimes [30,44]. We recommend a wider application of DNA barcoding for identification of orchid species in illegal export, as it enables identification of material that is unidentifiable by morphology alone.

\section{Endnote}

${ }^{\mathrm{a}}$ Footnote: author names are provided for all species in Table 1. Species not included in Table 1 have author names included in the manuscript body.

\section{Competing interests}

The author(s) declare that they have no competing interests.

\section{Authors' contributions}

$A S, B G$, and $R C$ conceived the research. $A S$ and $B K$ were responsible for field research and interviews. $A S, B K, B G$ and $R C$ identified the herbarium vouchers; AS, YC, YD, TA and HB processed the data and performed the quantitative analysis. AS, HB and BG contributed to the manuscript. All authors have read and approved the final manuscript.

\section{Acknowledgements}

We thank local plant healers, orchid collectors, vendors, traders and district forest offices of the study sites for providing information. Bijaya Pant, Bhakta Raskoti and Muna Udas are thanked for providing us with relevant literature. Financial support to Abishkar Subedi was provided by the Alberta Mennega Stichting and to Ram Chaudhary by NUFU (Norwegian Council for Higher Education's Programme for Development Research and Education and NoMA Norads' Program for Master Studies).

\section{Author details}

${ }^{1}$ Naturalis Biodiversity Center, Sylviusweg 72, P.O. Box 9517, Leiden, The Netherlands. ${ }^{2}$ Local Initiatives for Biodiversity, Research and Development (LI-BIRD), P.O. Box 324, Pokhara, Nepal. ${ }^{3}$ Central Department of Botany, Tribhuvan University, Kirtipur, Nepal. ${ }^{4}$ Institute Biology Leiden, Einsteinweg 55, 2333 CC Leiden, The Netherlands. ${ }^{5}$ Department of Organismal Biology, Uppsala University, Norbyvägen 18 D, SE-75236 Uppsala, Sweden.
Received: 26 July 2013 Accepted: 23 August 2013

Published: 31 August 2013

\section{References}

1. Lawler LJ: Ethnobotany of the Orchidaceae. In Orchid Biol Rev Perspect lii. Ithaca, NY \& London, UK: Comstock Publ. Associates; 1984:27-149.

2. Jalal JS, Kumar P, Pangtey YPS: Ethnomedicinal orchids of Uttarakhand. Western Himalaya. Ethnobot. Leafl 2008, 2008:164.

3. Singh A, Duggal S: Medicinal orchids-an overview. Ethnobot Leafl 2009, 2009:3.

4. Dhyani A, Nautiyal BP, Nautiyal MC: Importance of astavarga plants in traditional systems of medicine in Garhwal, Indian Himalaya. Int J Biodivers Sci Ecosyst Serv Manag 2010, 6:13-19.

5. Hossain MM: Therapeutic orchids: traditional uses and recent advancesan overview. Fitoterapia 2011, 82:102-140.

6. Raskoti BB: The orchids of Nepal. Kathmandu, Nepal: Quality printers; 2009.

7. Manandhar NP: Plants and people of Nepal. Portland, OR: Timber Press; 2002.

8. Shrestha KK, Rajbhandary S, Tiwari NN, Poudel RC, Uprety Y: Ethnobotany in Nepal: review and perspectives. Wwf Nepal Program: Kathmandu Nepal; 2004.

9. Acharya KP, Rokaya MB: Medicinal orchids of Nepal: are they well protected? Our Nat 2010, 8:82-91.

10. Subedi A: Orchids around Pokhara valley of Nepal. Local Initiatives for Biodiversity, Research and Development: Pokhara, Nepal; 2002.

11. Vaidya BN, Shrestha M, Joshee N: Report on Nepalese orchids species with medicinal properties. In The Himalayan plants, can they save us? Proceeding of Nepal-Japan joint symposium on conservation and utilization of Himalayan medicinal resources. Edited by Watanabe T, Takano A, Bista MS, Saiju HK. Japan: Society for the Conservation and Development of Himalayan Medicinal Resources (SCDHMR); 2002:146-152.

12. Bhattarai S, Chaudhary RP, Taylor RSL: Prioritization and trade of ethnomedicinal plants by the people of Manang district, central Nepal. In Veg. Soc. their interact: Himalayas. Edited by Chaudhary RP, Subedi BP, Vetaas O. Norway: Tribhuvan University, Nepal and University of Bergen; 2002:151-169.

13. Bailes CP: Orchids in Nepal, the conservation and development of a natural resource, advisory report and recommendations. Richmond, UK: Royal Botanic Gardens Kew; 1985.

14. Shakya LR, Bajracharya BM, Chettri MK: WWF Nepal Program Report Series 8. Conserving the threatened orchids of Kathmandu valley. WWF Nepal: Kathmandu, Nepal; 1994.

15. Wildlife Conservation Nepal: Orchids in the Churiya hills and their survival in Nepal. Kathmandu, Nepal: Wildlife Conservation Nepal; 2003.

16. King G, Pantling R: The orchids of the Sikkim-Himalaya. Bengal Secretariat Press: Calcutta; 1898.

17. White KJ, Sharma B: Wild orchids in Nepal: the guide to the Himalayan orchids of the Tribhuvan Rajpath and Chitwan jungle. Bangkok, Thailand: White Lotus Press; 2000.

18. Pearce NR, Cribb PJ: The orchids of Bhutan: flora of Bhutan: vol. 3, part 3. Royal Botanic Garden Edinburgh and Royal Government of Bhutan, The Charlesworth Group: Huddersfield, U.K; 2002.

19. Balami NP: Ethnomedicinal uses of plants among the Newar community of Pharping village of Kathmandu district. Nepal Tribhuvan Univ J 2004, 24:13-19.

20. Chen Y, Xu J, Yu H, Qing C, Zhang Y, Liu Y, Wang J: 3,7-Dihydroxy-2,4,6trimethoxyphenanthrene, a new phenanthrene from Bulbophyllum odoratissimum. J-Korean Chem Soc 2007, 51:352.

21. Pyakurel D, Gurung K: Enumeration of orchids and estimation of current stock of traded orchids in Rolpa district: final report. Rolpa, Nepal: District forest office Rolpa; 2008.

22. Thakur RB, Yadav RP, Thakur NP: Enumerating the status of orchid species of Makawanpur district. Hamro Kalpabricha 2010, 20:1-18.

23. Gewali MB: Aspects of traditional medicine in Nepal. Toyama, Japan: Inst. Nat. Med. Univ. Toyama; 2008.

24. Lama YC, Ghimire SK, Aumeeruddy-Thomas Y: Medicinal plants of Dolpo: Amchis' knowledge and conservation. Kathmandu, Nepal: WWF; 2001.

25. Nepal IUCN: National register of medicinal \& aromatic plants. Kathmandu, Nepal: IUCN Nepal Country Office for His Majesty's Government of Nepal, Ministry of Forests and Soil Conservation; 2004

26. Tezuka Y, Ji L, Hirano H, Ueda M, Nagashima K, Kikuchi T: Studies on the constituents of orchidaceous plants IX constituents of Spiranthes sinensis (Pers.) Ames var. amoena (M. Bieberson [M. Bieberstein]) Hara.(2): 
structures of spiranthesol, spiranthoquinone, spiranthol- $C$ and spiransineol-B, new isopentenyldihydrophenanthrenes. Chem Pharm Bull (Tokyo) 1990, 38:629-635.

27. Eurlings MCM, Gravendeel B: TrnL-trnF sequence data imply paraphyly of Aquilaria and Gyrinops (Thymelaeaceae) and provide new perspectives for agarwood identification. Plant Syst Evol 2005, 254:1-12.

28. Eurlings MCM, Heuveling van Beek H, Gravendeel B: Polymorphic microsatellites for forensic identification of agarwood (Aquilaria crassna). Forensic Sci Int 2010, 197:30-34

29. Ogden R, Dawnay N, McEwing R: Wildlife DNA forensics—bridging the gap between conservation genetics and law enforcement. Endanger Species Res 2009, 9:179-195.

30. Dawnay N, Ogden R, McEwing R, Carvalho GR, Thorpe RS: Validation of the barcoding gene $\mathrm{COI}$ for use in forensic genetic species identification. Forensic Sci Int 2007, 173:1-6.

31. Gravendeel B, Chase MW, De Vogel EF, Roos MC, Mes TH, Bachmann K: Molecular phylogeny of Coelogyne (Epidendroideae; Orchidaceae) based on plastid RFLPs, matK, and nuclear ribosomal ITS sequences: evidence for polyphyly. Am J Bot 2001, 88:1915-1927.

32. Altschul SF, Gish W, Miller W, Myers EW, Lipman DJ: Basic local alignment search tool. J Mol Biol 1990, 215:403-410.

33. Gravendeel B, Eurlings M, van den Berg C, Cribb PJ: Phylogeny of Pleione (Orchidaceae) and parentage analysis of its wild hybrids based on plastid and nuclear ribosomal ITS sequences and morphological data. Syst Bot 2004, 29:50-63.

34. Subedi A: Orchids and sustainable livelihoods: initiatives in Nepal to manage globally threatened biodiversity. In Proc. 18th world orchid conf. Edited by Raynal-Roques A, Roguenant A, Prat D. Turriers, France: Naturalia Publications; 2005:470-474.

35. Kong JM, Goh NK, Chia LS, Chia TF: Recent advances in traditional plant drugs and orchids. Acta Pharmacol Sin 2003, 24:7-21.

36. Maikhuri RK, Rao KS, Chauhan K, Kandari LS, Prasad P, Rajasekaran C: Development of marketing of medicinal plants and other forest products: can it be a path way for effective management and conservation? Indian For 2003, 129:169-178.

37. Kalimuthu K, Senthilkumar R, Vijayakumar S: In vitro micropropagation of orchid, Oncidium sp. (dancing dolls). Afr J Biotechnol 2007, 6:1171-1174.

38. Chugh S, Guha S, Rao IU: Micropropagation of orchids: a review on the potential of different explants. Sci Hortic 2009, 122:507-520.

39. Swar S: Micropropagation of Cymbidium iridoides D. Don and Coelogyne cristata Lindl. (Orchidaceae). MSc thesis. Tribhuvan University: Central Department of Botany; 2003.

40. Gurung R: In vitro propagation of Aerides odorata Lour. by shoot tip culture. MSc thesis. Tribhuvan University: Central Department of Botany; 2005.

41. Shrestha A: Ex situ conservation of Coelogyne ovalis Lindl. (Orchidaceae) through micropropagation. MSc thesis. Tribhuvan University: Central Department of Botany; 2005.

42. Coghlan M, Haile J, Houston J, Murray D, White N, Moolhuijzen P, Bellgard M, Bunce M: Deep sequencing of plant and animal DNA contained within traditional Chinese medicines reveals legality issues and health safety concerns. Plos Genet 2012, 8:e1002657.

43. Kool A, De Boer HJ, Krüger A, Rydberg A, Abbad A, Björk L, Martin G: Molecular identification of commercialized medicinal plants in Southern Morocco. Plos One 2012, 7:e39459.

44. Wasser SK, Mailand C, Booth R, Mutayoba B, Kisamo E, Clark B, Stephens M: Using DNA to track the origin of the largest ivory seizure since the 1989 trade ban. Proc Natl Acad Sci 2007, 104:4228.

\section{Submit your next manuscript to BioMed Central and take full advantage of:}

- Convenient online submission

- Thorough peer review

- No space constraints or color figure charges

- Immediate publication on acceptance

- Inclusion in PubMed, CAS, Scopus and Google Scholar

- Research which is freely available for redistribution

Submit your manuscript at www.biomedcentral.com/submit 